\title{
Study on the Audiovisual Language Style of Chinese Animation Works
}

\author{
Liming Liu \\ Zhuhai College of Jilin University, Zhuhai, Guangdong, 519041
}

Keywords: Audiovisual Language Style, Chinese Animation, Television Development

\begin{abstract}
Animation, as an art form, with the development of society and the advancement of science and technology, its application field is more and more extensive. As a branch of the film, it is called by many people as the "ninth art". In the glorious period of China's animation industry, in the past few decades after the founding of the People's Republic of China, the art of audio-visual language of domestic animation has fully utilized the art form of the nation, and formed an aesthetic style and creative mode with Chinese national characteristics in the process of exploring the style of national form.
\end{abstract}

\section{Introduction}

With the continuous development of the cultural industry, people need to seek more spiritual pleasure. The cartoons are developed in accordance with the trend of this era. Whether it is the development of the animation industry or the spread of national culture, it depends on the carrier of cartoon audiovisual language. The art of audio-visual language is the basis for the realization of animation. Therefore, the development of the style of audio-visual language is a key factor in the development of animation. From the American Mickey Mouse to the Japanese Miyazaki "Spirited Away", various styles of cartoons have fully demonstrated its great artistic charm. It brings great visual impact to people and also gives people great spiritual enjoyment and improvement of ideological realm. Animation art can no longer exist only as an aesthetic form. It also bears multiple responsibilities such as creating a box office, raising ratings, stimulating the economy, promoting culture, and promoting ideas. Driven by various interests, it is not difficult to see that all countries are making efforts to promote the development of their own animation. Appreciators' evaluation of the work is the key to judging the success of an animation work. Therefore, the recognition of the audience, the perfect expression of art and the embodiment of the national style are extremely important. Xu Mougeng once thought: "Our ancients have long said that people's eyes and ears are the same. Just because people's audiovisuals will pass, they always hope to use them together, eyes and noses, and the sense organs work closely together to achieve a comprehensive Sensory enjoyment." (1) Simultaneous animation as a branch of the film, the organic combination of audiovisual language, is even more important. It can be said that without the expression of audiovisual language, animation will not be able to show its artistic charm.

\section{Animated audiovisual language style and its importance}

We define style as the character and temperament of a work in terms of content, vision and hearing. It is the best artistic carrier for distinguishing works and creators. Thus it reveals the connection between artistic creation and artistic appreciation. In animation, the comprehensive performance of audiovisual language is the most important part of an animated cartoon. Its external stylistic features are the most subjective and direct way to judge an animation. Different art categories have different requirements for style, and the complicated language features are not only the key factors for forming style, but also the important externalization of style. The expression of language style is an important means for the work to convey artistic ideas, thoughts and feelings. Therefore, almost all directors regard the overall style of the cartoon audiovisual language as the top priority of the creative animation, which is the top priority after completing the script. Only when the style is determined can an animation be completed in one go, and vice versa, it will be 
intermittent, fragmented, and lose the whole. "The essence of style is also that it is the result of the artist's unique and vivid expression of the aesthetic object, and it is also the result of the artist's correct appreciation, experience and taste of the art, so it is in a more profound sense. It reveals one of the essential characteristics of artistic creation and appreciation: the infinite richness and diversity of the real world and aesthetic objects. The animated audiovisual language with different styles not only attracts viewers of different levels and ages, but also reflects the understanding and aesthetic preferences of each animation director for art, music, film, literature and other related culture and art. The animation language of modern society is no longer an art created for art. Its style is influenced by the commodity economy. Animation has become the aesthetic art of the public. The audience's favorite is the best embodiment of the success of animation works.

\section{Influencing factors of the development of domestic animation audio-visual language style}

UNESCO defines the cultural industry as "a series of activities that produce, produce, store and distribute cultural goods and services in accordance with industrial standards. Its main purpose is not to promote greater cultural development but to pursue economic benefits”. . Although this statement is not proper, we must admit that animation has been inextricably linked with the development of the economy since its birth. If the development of science and technology is its embryo, then economic development is to promote the development of animation style. Nutrients. In the market economy, animation has two attributes as a cultural industry: one is its artistic attributes, and the other is its products and commodity attributes. In the final analysis, artistry is the foundation, and commodityity is the purpose and motivation. Market-oriented animation is not life and long-term development. The audiovisual language style of animation directly affects the attention of the work and the revenue of the box office. Therefore, for the purpose of commercial animation, we must pay more attention to the audience's feelings. The artistic language form must be in line with the public's aesthetics, which can attract people's attention. It is an artistic language style that can be used by the general public to create box office income. . This is why more and more cartoons in the past few years have made the scene performance and realistic visual effects the main style of their audio-visual language, and they have invested millions of dollars to improve the audio-visual experience of cartoons. I don't mean to ask the market, not art, but the market demand has a guiding effect on the style of animated audio-visual language. Good animation works can bring both benefits and artistic charm of animation, and there is no style. The fascinating animation works are unlikely to bring good economic benefits.

\section{Visual language development in domestic animation}

Folk art is a spiritual material product created and enjoyed by the people in their lives. It comes from the collective aesthetic consciousness formed by the specific social structure and cultural structure of rural China. It maintains more originality and has a great Spontaneous, amateur and entertaining are closer to the material life of reality and have a clear utilitarian meaning. This is the so-called folk culture or folk culture. For the image carrier of this culture, we call it folk art. The folk art in the styling has a strong sense of decoration, the concept is very clever, the imagination is bold, exaggerated, and very romantic. As shown in the picture: the art of paper-cutting, using the hollow carving to show the shape of people or objects, the patterns are mostly central symmetry and symmetrical figures; the shadow art style is mostly "side image", the stylized features are prominent, the characters are only active at the joint position. , to form a unique sports mode; new year paintings or folk paintings, full of pictures, rich characters, and mostly children. These characteristics coincide with the image of animation art.

\section{Domestic animation and audio-visual language style development and reference}

Today's audio-visual language style is inseparable from the public aesthetic, so entertainment is also an indispensable factor in commercial animation. As the leading player in the animation 
industry today, the United States, whether it is Disney, DreamWorks or Pixar, is market-oriented, so its style is exaggerated and entertaining. For example, the "Monster Company" produced by American Disney Animation is very comical and entertaining. The plot is easy and humorous, the scene is very fantasy, and the picture color is saturated and intense. And the use of advanced animation virtual technology and 3D modeling production methods, strengthen the role of the sense of volume and texture. As shown in the picture, the intimidating expert Sullivan is a green-haired monster with purple spots. The body is tall and strong, but not terrible. Its big eyes are full of love, and the two horns on the top of the head are also very cute. . Unlike Sullivan, Monster Mike's design is extremely simple. The nearly oval body has only one big eye and one big mouth. A pair of small horns don't seem to be fully developed. With thin arms and thin legs, it's very funny. . Other monster characters are also innovative and refreshing.

Japanese cartoons are different from the United States. The styles are not based on exaggerated distortions. Most of them use realistic writing techniques with a little landscaping. Therefore, it is not difficult to find that most of the Japanese animated characters have the same slender figure, nine-headed body, pointed chin, big eyes, small mouth and nose, and a beautiful route. "Sailor Moon", "Ohran University College Men's Public Relations Department", "Fruit Basket", etc., although each animation works differs in style because of different themes and directors, it still makes people see this at a glance. It is Japanese animation. In addition to deliberately increasing the proportion of legs and eyes, Japan prefers the use of "symbols" in character styling, such as the symbolic signs of ninjas in Naruto. A symbol represents a character attribute, which not only has characteristics in the image, but also increases the viewer's awareness and forms a default visual image. In addition to character modeling, the scene design of Japanese animation is also very different from other national animations. Whether it is hand-painted or computer-drawn, Japanese animation scenes are characterized by simplicity, realism and sophistication.

In recent years, the character style has become the new favorite of TV screens. The "version" is an exaggerated style that shortens the proportion of facial features. It originated from Japanese comic characters and has been sought after by Korean animation. Now it has become one of the important expressions of commercial animation. Japanese animation "In the performance of characters, there are often two kinds of beautification and drama (ie, edition). The two characters appear alternately, showing different moods of the characters, which not only enriches the picture, but also adds a sense of humor and psychological emotion. The appeal of this." South Korea even directly used this styling as an animated character, making "” everywhere, greatly expanding the scope of use. The style of the character is smart and cute, with strong affinity and fun, and is widely accepted and loved by the public. It has become a transcript of many animated character designers. The overall character of the version of the character is the head and body, the limbs are short and obese, and the height is generally between the heads. Based on the facial features of infants and children, it is simplified, deformed and exaggerated, with watery eyes, small nose and mouth. The expression is exaggerated and bold, and has a strong performance. In our cartoons, the Chinese version of the art of modeling is also often used, and has achieved good results. Such as the animated film "Three Kingdoms", "Large Li Bai", "Pleasant Goat and Big Big Wolf" and so on. In particular, the image in "Pleasant Goat and Big Big Wolf" has been deeply rooted in the hearts of the people. The smart and cute of the Pleasant Goat, the lively and playfulness of the beautiful sheep, the clumsyness of the lazy sheep, the timid obedience of the Gray Wolf, the rudeness of the Red Wolf, not only by the children. Welcome, many adults also like these lovely images. Therefore, good learning and open-minded learning are very important for the development of domestic animation.

\section{Conclusion}

In order to keep up with the world animation track, the creation of Chinese cartoons must be based on local art, actively absorb nutrition from national culture, use advanced science and technology, grasp the pulse of the times, and strive to explore the style, form and production method of animation and audio-visual language art. In order to present Chinese art and Chinese culture to 
the audience in the media of animation. Produce a real animation work that is "educational, fun, and fun”. The author believes that as long as we make unremitting efforts, Chinese animation art will surely become a new noble in the world of animation.

\section{References}

[1] Wang Daguo. The basic characteristics of TV documentary [J]. News enthusiasts. 2010.21.

[2] Zhao Zhipeng. Analysis of the development of contemporary Chinese TV documentary column [D]. Hebei University. 2009.

[3] Zhang Xiu. Analysis of the status quo and development prospects of TV documentary columns [J]. China TV. 2009.2.

[4] Liu Shuyan. Reflections on the survival and development of documentary columnarization [J]. Contemporary TV. 2010.3. 